\title{
PIV measurement of complex flow characteristics in open-cell metal foam replica
}

\author{
Minsin Kim ${ }^{1}$, Youngwoo Kim ${ }^{1}$, Sajjad Hosseini ${ }^{1}$, Kyung Chun Kim ${ }^{1 *}$ \\ ${ }^{1}$ Pusan National University, School of Mechanical Engineering, Busan, South Korea \\ *kckim@pusan.ac.kr
}

\begin{abstract}
Time-resolved 2-D particle image velocimetry was used to study on turbulent flow characteristics inside an open-cell metal foam under the laminar and turbulent inlet conditions. A study on the effect of Reynolds number was conducted with different three channel Reynolds numbers, 1000, 5000 and 10000. Uniform upstream flow is divided by the pore network of metal foam and it is found that there are flow disturbances induced by metal foam structure even at a laminar inlet condition. It is confirmed that there is a similarity of the preferred flow path flows take regardless of Reynolds number.
\end{abstract}

\section{Introduction}

Metal foam is a metallic sponge which consists of many ligaments and nodes interconnect the struts. Metal foam structures have been of interest because their geometrical characteristics like high porosity, large specific surface area. Due to the great potential of applications, various studies have been conducted to understand the flow inside metal foam. However, flow characteristics inside metal foam are not fully understood because highly complex geometry of metal foam makes us difficult to observe flow inside metal foam. The characteristics of metal foam internal flow with a laminar flow inlet condition were studied by Moon et al. (2018). In the present study, however, particle image velocimetry (PIV) measurement was conducted to observe the turbulent characteristics of inside metal foam in detail when turbulent flow or laminar flow enters the metal foam replica.

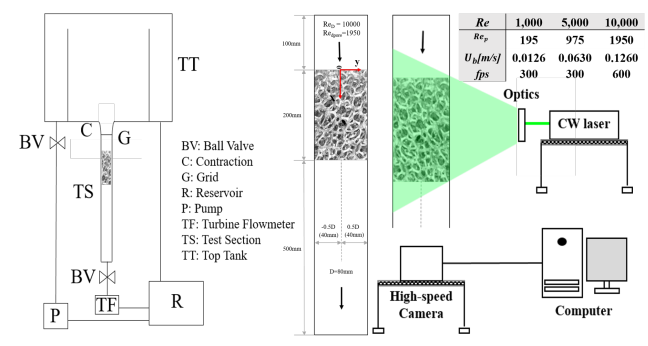

Figure 1: Experimental setup

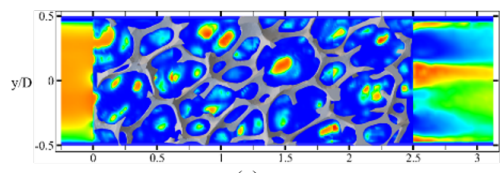

(a) $\times / \mathrm{D}$

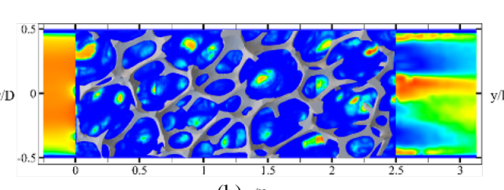

(b) $\mathrm{x} / \mathrm{D}$

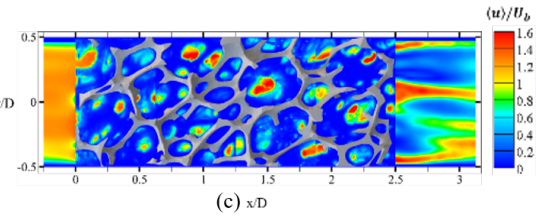

(c) $\times \mathrm{D}$

Figure 2: Contours of mean velocity magnitude of 6 view (a) $\operatorname{Re}=10,000$ (b) $\operatorname{Re}=5,000$ (c) $\operatorname{Re}=1,000$ 

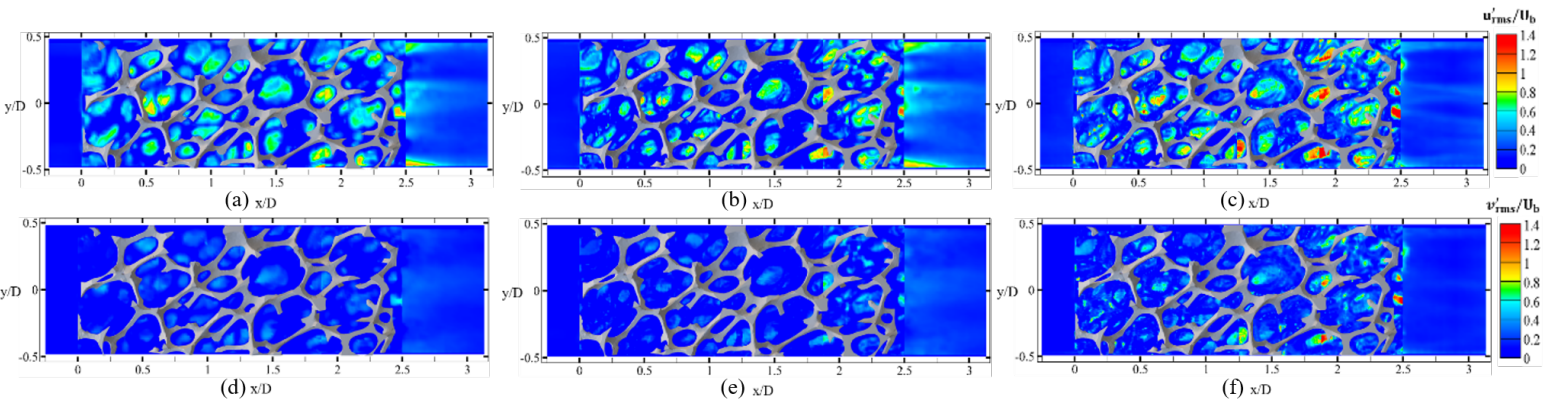

Figure 3: Contours of root-mean-squared temporal fluctuation velocities of streamwise component (upper row) and transverse component (lower row) (a),(d) $\operatorname{Re}=10,000$ (b),(e) $\operatorname{Re}=5,000$ (c), (f) $\operatorname{Re}=1,000$

\section{Method}

The size of the replica is $80 \mathrm{~mm} \times 80 \mathrm{~mm} \times 200 \mathrm{~mm}$ and the cell diameter, pore diameter, strut diameter, and porosity of the replica are $36.08 \pm 4.24 \mathrm{~mm}, 15.6 \pm 1.32 \mathrm{~mm}, 3.2 \pm 0.24 \mathrm{~mm}$, and 0.92 , respectively. The transparent metal foam model was placed inside a vertical water channel with same dimension. The experimental loop containing the test section is shown in Fig. 1.

\section{Results and Discussion}

Fig. 2 2 shows the velocity magnitude fields. Refractive index of metal foam and fluid could not be matched, however, it was possible to observe the flow in part due to the large size of the pore. It is found that there is similarity in the flow path regardless of Reynolds number. This indicates that the preferred path the fluid takes through the metal foam structure is not different in the different flow regimes. This is similar result from Lu et al. (2020). Fig. 3 shows contours of the fluctuation velocities. When a turbulent flow with a turbulence intensity of $10 \%$ enters the metal foam, the turbulent intensity increases while passing through the metal foam replica. In the case of laminar inlet condition, the value of $u_{r m s}^{\prime} / U_{b}$ of the upstream are near zero, but they increase to 0.1-1.4 inside the metal foam structure. This indicates there are flow disturbances induced by metal foam structure even at a laminar inlet condition. After passing through the metal foam structure, turbulent decays due to the shear-free uniform flow and all the enhanced turbulence recover to initial state.

\section{Conclusions}

Further discussion about the turbulent characteristics such as vorticity and turbulent kinetic energy inside metal foam will be provided at the conference.

\section{Acknowledgements}

This work was supported by the National Research Foundation of Korea(NRF) grant funded by the Korea government(MSIT) (No. 2020R1A5A8018822).

\section{References}

Lu X, Zhao Y, and Dennis DJ (2020) Fluid flow characterisation in randomly packed microscale porous beds with different sphere sizes using micro-particle image velocimetry. Experimental Thermal and Fluid Science 118:110136

Moon C, Kim HD, and Kim KC (2018) Kelvin-cell-based metal foam heat exchanger with elliptical struts for low energy consumption. Applied Thermal Engineering 144:540-550 